\title{
Cold or Warm Dark Matter?: A Study of Galaxy Stellar Mass Distributions
}

\author{
Bruce Hoeneisen \\ Universidad San Francisco de Quito, Quito, Ecuador \\ Email: bhoeneisen@usfq.edu.ec
}

How to cite this paper: Hoeneisen, B. (2020) Cold or Warm Dark Matter?: A Study of Galaxy Stellar Mass Distributions. International Journal of Astronomy and Astrophysics, 10, 57-70. https://doi.org/10.4236/ijaa.2020.102005

Received: March 4, 2020

Accepted: March 31, 2020

Published: April 3, 2020

Copyright ( 2020 by author(s) and Scientific Research Publishing Inc. This work is licensed under the Creative Commons Attribution International License (CC BY 4.0).

http://creativecommons.org/licenses/by/4.0/

\begin{abstract}
We compare the observed galaxy stellar mass distributions in the redshift range $0<z \lesssim 11$ with expectations of the cold $\Lambda \mathrm{CDM}$ and warm $\Lambda \mathrm{WDM}$ dark matter models, and obtain the warm dark matter cut-off wavenumber: $k_{\mathrm{fs}}=0.90_{-0.34}^{+0.44} \mathrm{Mpc}^{-1}$. This result is in agreement with the independent measurements with spiral galaxy rotation curves, confirms that $k_{\mathrm{fs}}$ is due to warm dark matter free-streaming, and is consistent with the scenario of dark matter with no freeze-in and no freeze-out. Detailed properties of warm dark matter can be derived from $k_{\mathrm{fs}}$. The data disfavors the $\Lambda$ CDM model.
\end{abstract}

\section{Keywords}

Dark Matter, Warm Dark Matter, Dark Matter Properties, Galaxy Stellar Mass

\section{Introduction}

Most current cosmological observations are well described by the cold dark matter $\Lambda \mathrm{CDM}$ model with only six independent parameters, and a few assumptions that are consistent with present observations: flat space, a cosmological constant, and scale invariant adiabatic primordial density perturbations [1]. This economical description of the universe is apparently in agreement with all observations on large scales, but seems to have tensions with some small scale phenomena: the "cusp vs core" problem of spiral galaxies, i.e. simulations obtain a cusp while observations find a core, and the "missing satellite" problem [2]. The $\Lambda$ CDM model assumes that dark matter has a negligible free-streaming length. However, fits to spiral galaxy rotation curves obtain a non-negligible dark matter free-streaming length [3]. This free-streaming cuts off the power spectrum of linear density perturbations at a comoving wavenumber $k_{\mathrm{fs}}$. Adding this parameter to the $\Lambda \mathrm{CDM}$ model obtains the warm dark matter model $(\Lambda \mathrm{WDM})$. 
We compare the observed galaxy stellar mass distributions in the redshift range $0<z \lesssim 11$ with expectations of the cold and warm dark matter models, and obtain the cut-off wavenumber $k_{\mathrm{fs}}$. The notation and cosmological parameters are as in Reference [1].

The outline of this article is as follows. In Section 2 we obtain predictions, based on the Press-Schechter formalism, of the stellar mass distributions for the cold and warm dark matter models. This formalism is valid only at redshifts $z \gtrsim 5$ as discussed in Section 3. In Section 4 we present measurements of $k_{\mathrm{fs}}$ by comparing predictions with data in the redshift range $5.5 \lesssim z \lesssim 8.5$. Section 5 verifies the compatibility between predictions and the galaxy with largest observed spectroscopic redshift to date. We close with conclusions.

\section{Predictions of the Stellar Mass Distributions}

Let $P(k)$ be the power spectrum of linear density perturbations in the cold dark matter $\Lambda \mathrm{CDM}$ model as defined in Reference [4], Equation (8.1.42). $k$ is the comoving wavenumber. If dark matter is warm, $P(k)$ becomes replaced by $P(k) \tau^{2}\left(k / k_{\mathrm{fs}}\right)$, where $\tau^{2}\left(k / k_{\mathrm{fs}}\right)$ is a cut-off factor. The cut-off is due to freestreaming of the warm dark matter particles.

In Reference [3] we consider a step-function cut-off factor. In that approximation, the first galaxies to form have the transition mass

$$
M_{\mathrm{fs}}=\frac{4}{3} \pi r_{\mathrm{fs}}^{3} \Omega_{m} \rho_{\text {crit }},
$$

where $r_{\mathrm{fs}}=1.555 / k_{\mathrm{fs}}$. Galaxies with larger masses form bottom up by hierarchical clustering. Once saturation is reached, galaxies that would have formed with mass $M$ may "not fit", loose mass to neighboring galaxies, and collapse with mass less than $M$. These are stripped down galaxies, they populate all masses, and are the only galaxies that form with mass less than $M_{\mathrm{fs}}$ in the step function approximation [3].

In the present article we take

$$
\tau^{2}\left(k / k_{\mathrm{fs}}\right)=\exp \left(-k^{2} / k_{\mathrm{fs}}^{2}\right) .
$$

This smooth cut-off is approximately the Born approximation of the calculation presented in Reference [5]. The true cut-off factor has a longer tail at large $k$ than the Born approximation [5]. To study the effect of the tail, we also consider the cut-off factor

$$
\tau^{2}\left(k / k_{\mathrm{fs}}\right)= \begin{cases}\exp \left(-k^{2} / k_{\mathrm{fs}}^{2}\right) & \text { if } k \leq k_{\mathrm{fs}}, \\ \exp \left(-k^{2} / k_{\mathrm{fs}}^{2}\right) \cdot k / k_{\mathrm{fs}} & \text { if } k>k_{\mathrm{fs}} .\end{cases}
$$

All figures, except Figure 13, include the tail: its effect is relatively small.

As we shall see in the following, the smooth cut-off results in bottom up hierarchical clustering, as in the $\Lambda \mathrm{CDM}$ model, up to saturation at redshift $z \approx 5$, and thereafter seems to become dominated by the generation of stripped down galaxies. Irregular "clumpy galaxies", that resemble beads on filaments or sheets [6], that are dynamically unstable and break up, may also contribute to the galaxy stellar mass function [6] [7]. 
The mean of the square of the fractional mass fluctuation in a sphere of comoving radius $r_{0}=1.555 / k_{0}$ (smoothed by a gaussian window function), and mass $M \equiv 4 \pi r_{0}^{3} \Omega_{m} \rho_{\text {crit }} / 3$, at redshift $z$, is [4]

$$
\sigma^{2}(M, z)=\frac{f^{2}}{(2 \pi)^{3}(1+z)^{2}} \int_{0}^{\infty} 4 \pi k^{2} \mathrm{~d} k P(k) \exp \left(-\frac{k^{2}}{k_{\mathrm{fs}}^{2}}\right) \exp \left(-\frac{k^{2}}{k_{0}^{2}}\right),
$$

while density perturbations are still linear. For simplicity, we have assumed the cut-off factor (2). $f$ is a correction due to the cosmological constant; $f=1,1.257,1.275$ for $z=0,2,11$, respectively [4]. For $r_{0}=8 / h \mathrm{Mpc}$, $\sigma(8 \mathrm{Mpc} / h, 0) \equiv \sigma_{8}=0.815 \pm 0.009$ [1] is becoming non-linear at the present time. $\sigma_{8}$ fixes the normalization of (4).

The Press-Schechter stellar mass function [8] is obtained from (4) as follows. The mass fraction locked up in halos with mass greater than $M$ at redshift $z$ is identified with the probability that the relative fluctuation of mass $M$ exceeds 1.686:

$$
F(M, z)=\frac{1}{2} \operatorname{erfc}\left(\frac{v}{\sqrt{2}}\right),
$$

where $v \equiv 1.686 / \sigma(M, z)$. Then $-(\partial F(M, z) / \partial M) \mathrm{d} M$ is identified with the mass fraction in halos with masses between $M$ and $M+\mathrm{d} M$. This identification is valid so long as the galaxies do not break up, or loose mass to neighboring galaxies, and have time to cluster. The Press-Schechter stellar mass function is then obtained after some algebra, and the inclusion of a "fudge factor" 2 [8], justified in [9]:

$$
\frac{\mathrm{d} n}{\mathrm{~d} \ln M}=\frac{\rho_{m}}{M} \frac{\mathrm{d} \ln \left(\sigma^{-1}\right)}{\mathrm{d} \ln M} f_{\mathrm{PS}}(v),
$$

where

$$
f_{\mathrm{PS}}(v)=\sqrt{\frac{2}{\pi}} v \exp \left(-\frac{v^{2}}{2}\right)
$$

and $\rho_{m} \equiv \Omega_{m} \rho_{\text {crit }}$. Equation (7) is valid in the spherical collapse approximation. A calculation that takes into account the average ellipticity and prolateness of perturbations, is the ellipsoidal collapse approximation, pioneered by R.K. Sheth and G. Tormen [10] [11], that replaces $f_{\mathrm{PS}}(v)$ by $f_{\mathrm{EC}}(v)$ :

$$
f_{\mathrm{EC}}(v)=0.322\left[1+\tilde{v}^{-0.6}\right] f_{\mathrm{PS}}(\tilde{v}),
$$

with $\tilde{v}=v$. Good fits to simulations are obtained with $\tilde{v}=0.84 v$ [11]. The factor 0.84 depends on the algorithm used to identify the collapsed halos, e.g. on the "link length" of the "friends-of-friends" algorithm, and also on the simulation volume. We note that Equations (6), (7) and (8), have no free parameters, except $k_{\mathrm{fs}}$.

Figures 1-3 present galaxy stellar mass function calculations for the $\Lambda \mathrm{CDM}$ model, and for $\Lambda \mathrm{WDM}$ with $k_{\mathrm{fs}}=1.6 \mathrm{Mpc}^{-1}$ and $0.8 \mathrm{Mpc}^{-1}$, respectively. We have converted from the halo mass $M$ to the stellar mass $M_{s}$ as follows: $\log _{10} M_{s}=\log _{10} M-0.63 \pm 0.19$ [3]. This uncertainty should be kept in mind when comparing the figures with observations. 


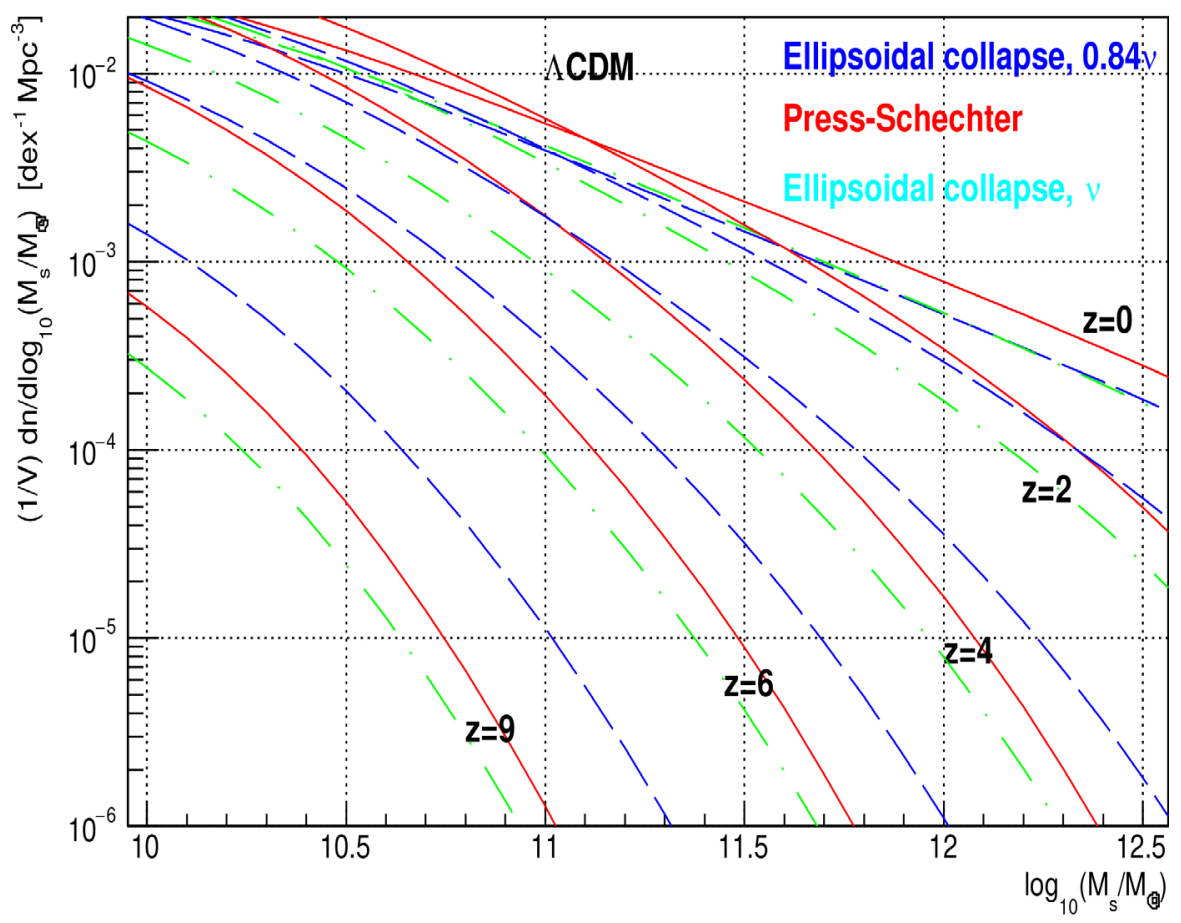

Figure 1. Calculated stellar mass functions with the Press-Schechter, Ellipsoidal Collapse with $\tilde{v}=v$, and Ellipsoidal Collapse with $\tilde{v}=0.84 v$, approximations, for the $\Lambda \mathrm{CDM}$ model, at several redshifts. These distributions are valid before saturation, i.e. for $z \gtrsim 5$.

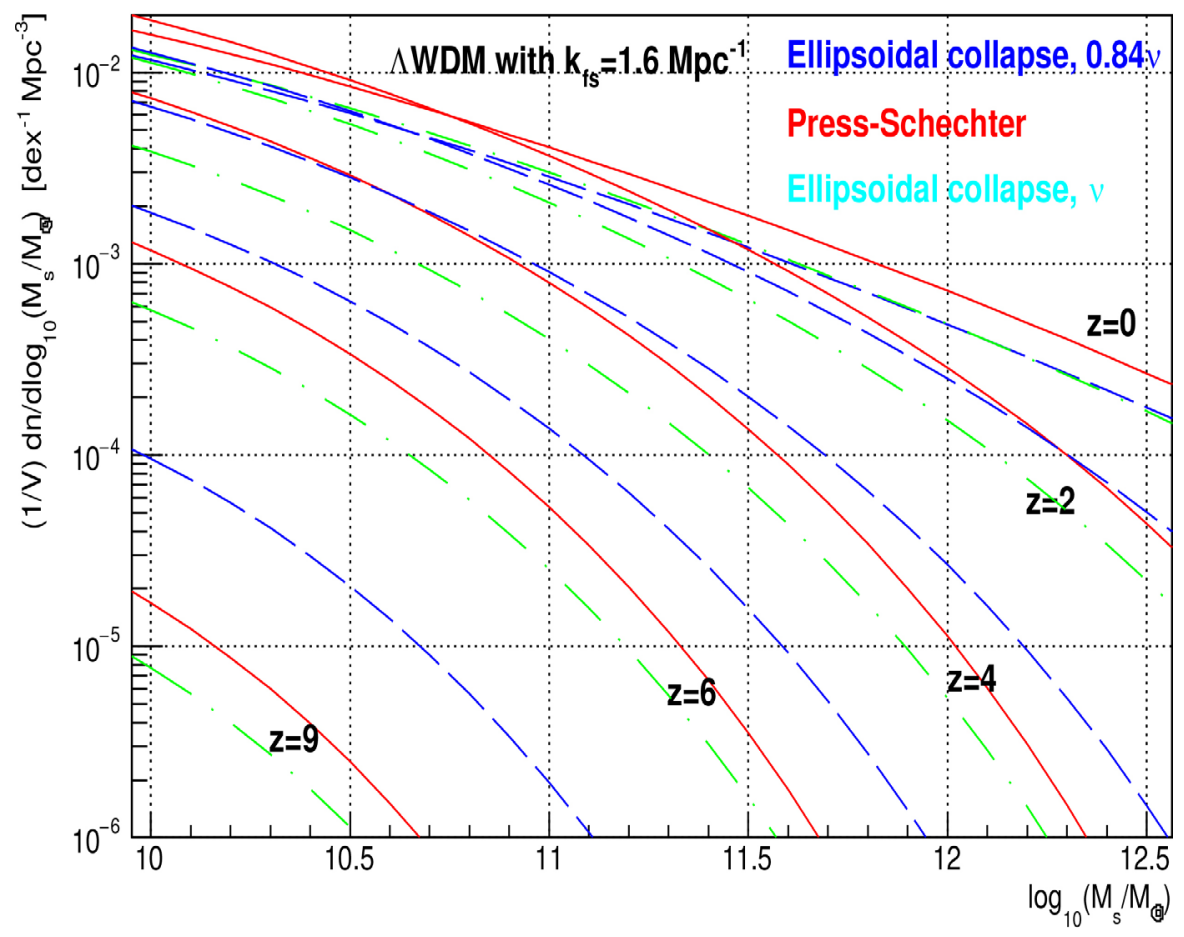

Figure 2. Calculated stellar mass functions with the Press-Schechter, Ellipsoidal Collapse with $\tilde{v}=v$, and Ellipsoidal Collapse with $\tilde{v}=0.84 v$, approximations, for the $\Lambda \mathrm{WDM}$ model with $k_{\mathrm{fs}}=1.6 \mathrm{Mpc}^{-1}$, at several redshifts. These distributions are valid before saturation, i.e. for $\mathrm{z} \gtrsim 5$. 


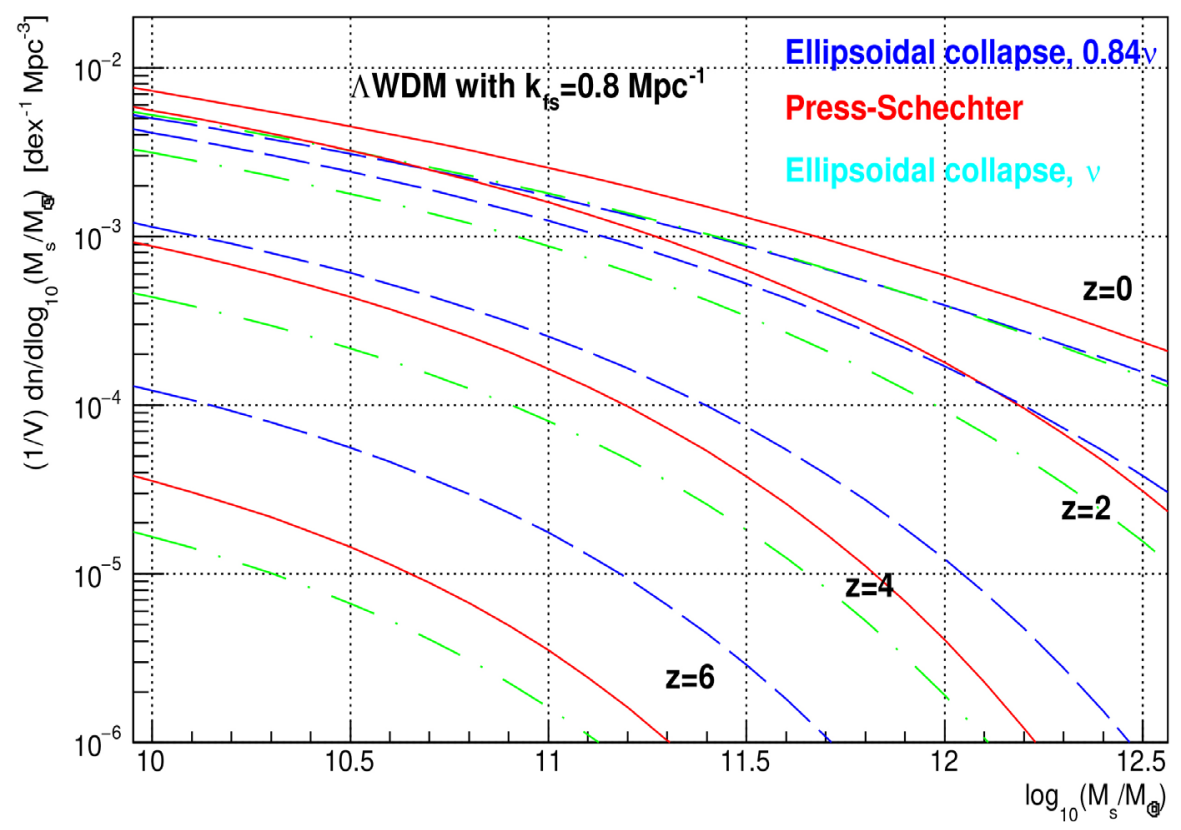

Figure 3. Calculated stellar mass functions with the Press-Schechter, Ellipsoidal Collapse with $\tilde{v}=v$, and Ellipsoidal Collapse with $\tilde{v}=0.84 v$, approximations, for the $\Lambda \mathrm{WDM}$ model with $k_{\mathrm{fs}}=0.8 \mathrm{Mpc}^{-1}$, at several redshifts. These distributions are valid before saturation, i.e. for $\mathrm{z} \gtrsim 5$.

\section{The Stellar Mass Distribution from SDSS Data}

We analyze Sloan Digital Sky Survey (SDSS) data release DR16 [12]. We include all data in the right ascension range $145^{\circ}$ to $230^{\circ}$, and declination range $0^{\circ}$ to $50^{\circ}$. By eye inspection of each redshift bin of this sky patch, we see only mild extraneous features such as zones with different exposure. The galaxy properties, including stellar mass, stellar age, star formation rate (SFR), and metallicity, are obtained from the photon spectra in filters $\mathrm{u}, \mathrm{g}, \mathrm{r}$, and $\mathrm{i}$, by several stellar population synthesis (SPS) models. The results that we analyze are placed in the following SDSS DR16 classes: stellarMassFSPSGranWideDust [13], stellarMassStarformingPort [14], stellarMassPCAWiscBC03 [15], and stellarMassPCAWiscM11 [15]. The SPS of these classes are described in the cited references. The galaxy stellar mass distributions for these SPS are presented in Figures 4-7, for several redshift bins. The units are counts per unit $\log _{10}\left(M_{s} / M_{\odot}\right)$ (dex) and unit comoving volume $\left(\mathrm{Mpc}^{3}\right) . M_{s}$ is the galaxy stellar mass returned by the SPS. The reduction of the distributions at low mass are due to the relative luminosity threshold of the observations. To obtain the galaxy stellar mass functions it is still necessary to divide by the stellar mass completeness factor (which is over $80 \%$ at $z<0.6$, and decreases at higher $z[16])$.

In Figure 4 we observe mass distributions that increase with redshift $z$ at the high mass end. This top down evolution is also observed by the Dark Energy Survey (DES), see Figure 7 of Reference [17]. If we assume that the mass corresponding to a threshold factor $1 / 2$ scales as the square of the luminosity distance, then the shift of the distributions to the right for $0.4<z<0.7$ should be even larger. 


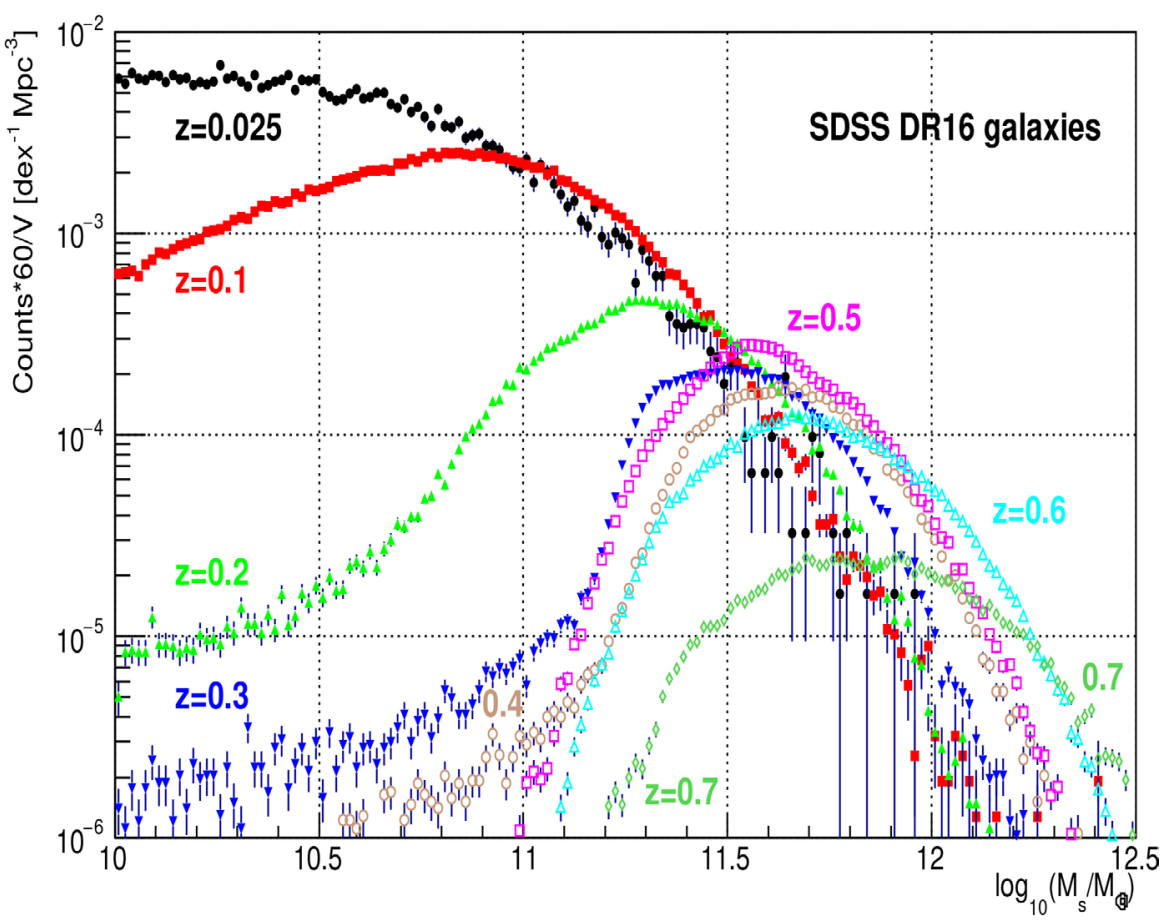

Figure 4. Galaxy counts per dex per comoving volume $\mathrm{d} n / \mathrm{d} \log _{10}\left(M_{s} / M_{\odot}\right) V \quad\left[\mathrm{dex}^{-1} \mathrm{Mpc}^{-3}\right]$ as a function of $\log _{10}\left(M_{s} / M_{\odot}\right)$ from SDSS DR16 data in class stellarMassFSPSGranWideDust [13], in bins of redshift $z$ of width \pm 0.5 (bin $z=0.025$ has width \pm 0.025 ).

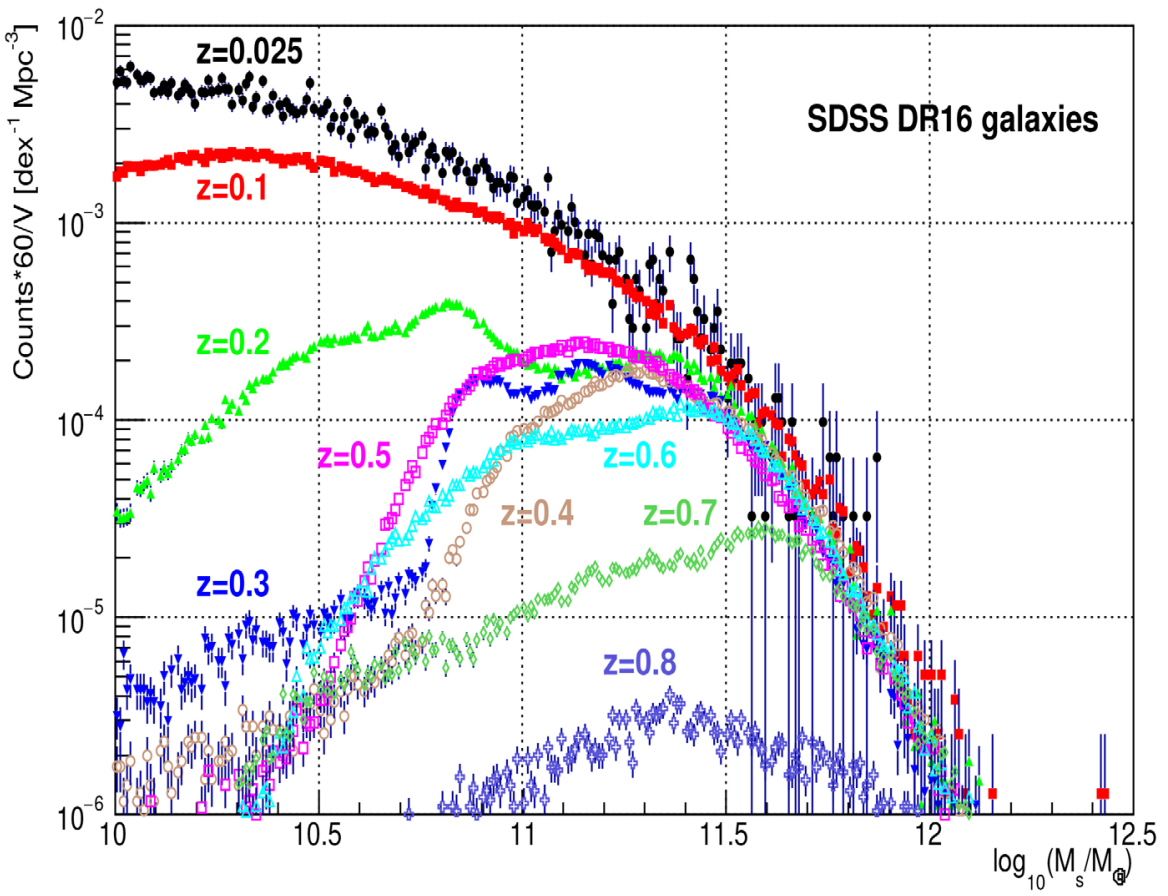

Figure 5. Galaxy counts per dex per comoving volume $\mathrm{d} n / \mathrm{d} l o g_{10}\left(M_{s} / M_{\odot}\right) V \quad\left[\mathrm{dex}^{-1} \mathrm{Mpc}^{-3}\right]$ as a function of $\log _{10}\left(M_{s} / M_{\odot}\right)$ from SDSS DR16 data in class stellarMassStarformingPort [14], in bins of redshift $z$ of width \pm 0.5 (bin $z=0.025$ has width \pm 0.025 ). 


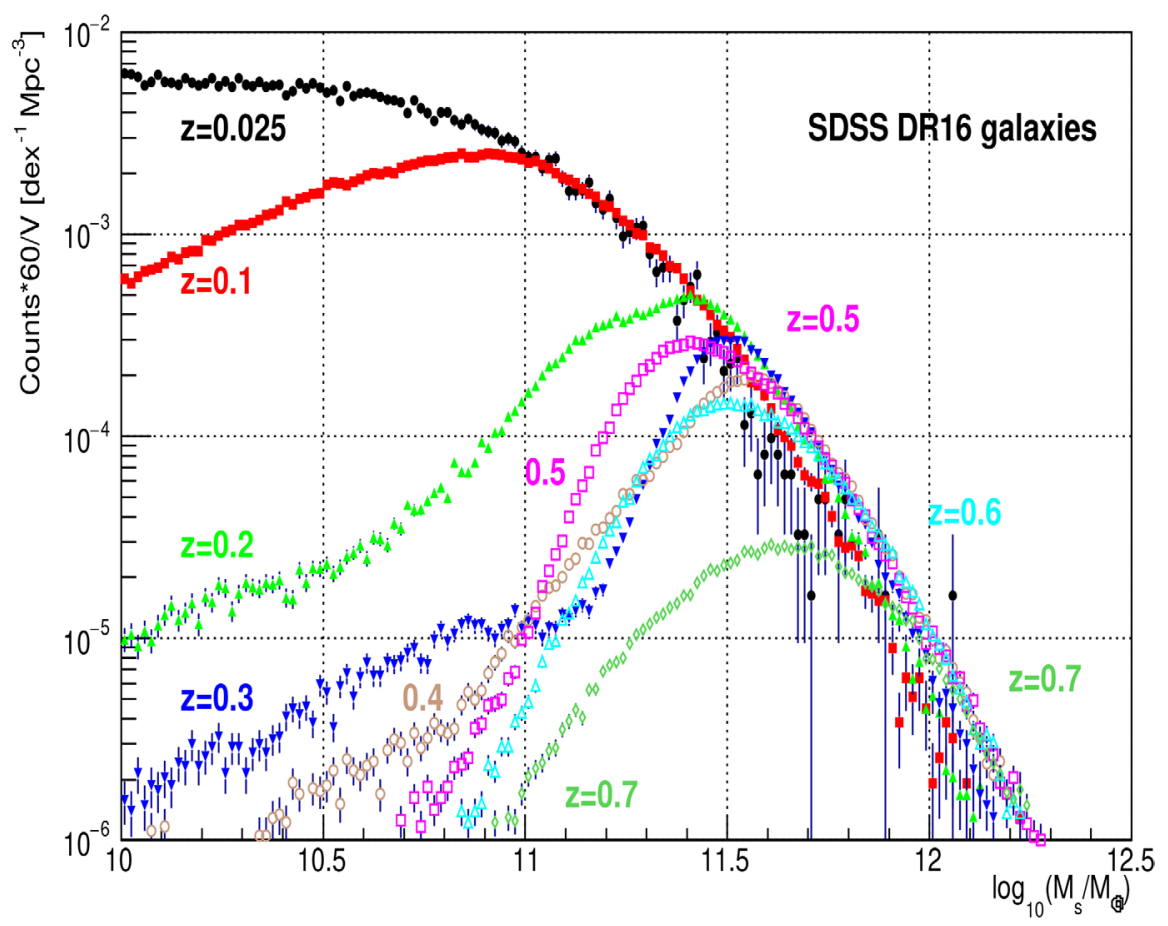

Figure 6. Galaxy counts per dex per comoving volume $\mathrm{dn} / \mathrm{d}_{\log }\left(M_{s} / M_{\odot}\right) V \quad\left[\mathrm{dex}^{-1} \mathrm{Mpc}^{-3}\right]$ as a function of $\log _{10}\left(M_{s} / M_{\odot}\right)$ from SDSS DR16 data in class stellarMassPCAWiscM11 [15], in bins of redshift $z$ of width \pm 0.5 (bin $z=0.025$ has width \pm 0.025 ).

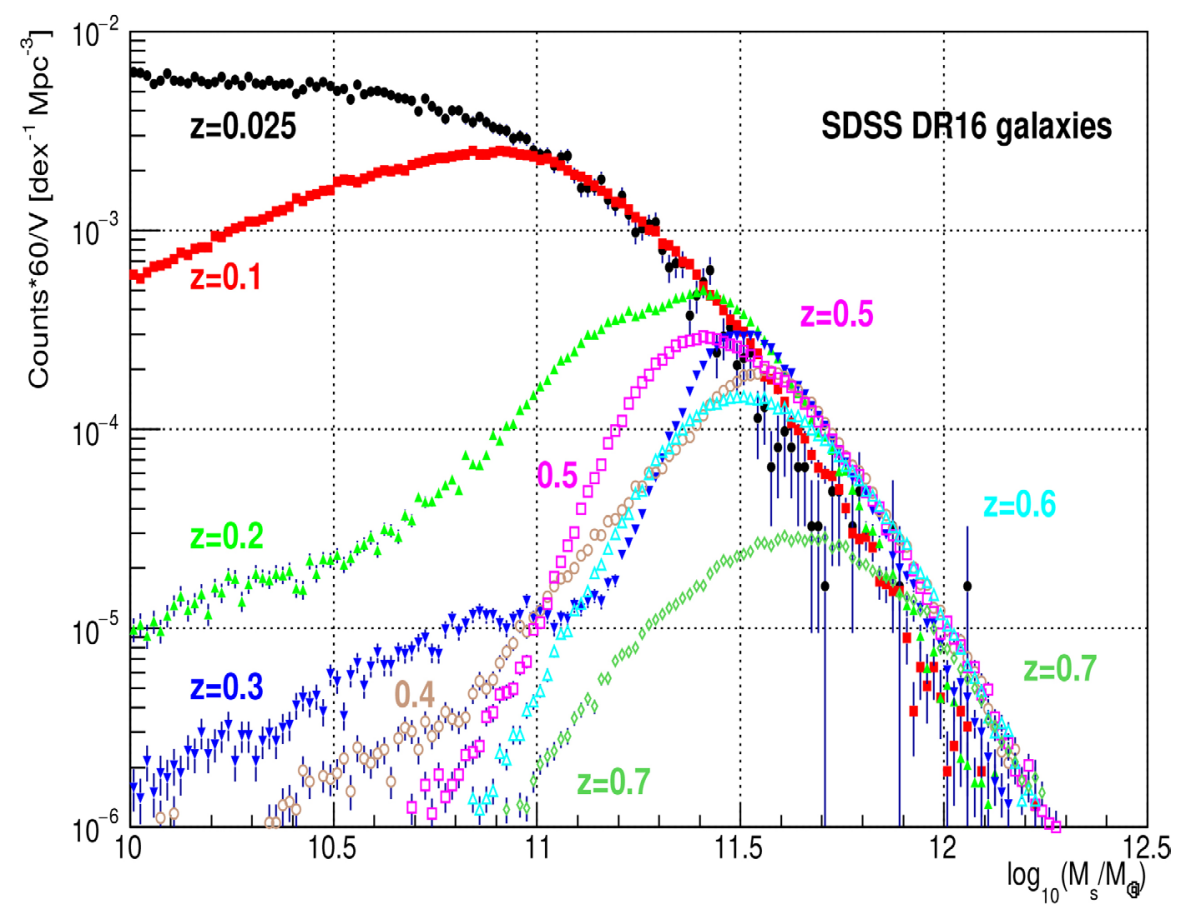

Figure 7. Galaxy counts per dex per comoving volume $\mathrm{dn} / \mathrm{d} \log _{10}\left(M_{s} / M_{\odot}\right) V \quad\left[\mathrm{dex}^{-1} \cdot \mathrm{Mpc}^{-3}\right]$ as a function of $\log _{10}\left(M_{s} / M_{\odot}\right)$ from SDSS DR16 data in class stellarMassPCAWiscBC03 [15], in bins of redshift $z$ of width \pm 0.5 (bin $z=0.025$ has width \pm 0.025 ). 
The top down evolution is observed even when the expected mass is replaced by the median mass minus one standard deviation, so the excess at high mass is not due to a statistical fluctuation. However, Figure 5 presents galaxy stellar mass distributions that do not change significantly with redshift. In Figure 6 and Figure 7 the evolution is slightly top down. In summary, at our current level of understanding, in the redshift range $0<z \lesssim 0.7$ the galaxy stellar mass function either evolves top down, or is stationary within observational uncertainties.

Let us compare the observed stellar mass function at $z=0$, e.g. Figure 4, with the calculations in Figures 1-3. We find that at $M_{s}=10^{12} M_{\odot}$ the calculations at $z_{\text {sat }} \approx 5$ already matches the observation at $z=0$. This "saturation" at the high mass end is not understood. At $M_{s}=10^{10} M_{\odot}$ we obtain $z_{\text {sat }}=7,4$ and 2 for $k_{\mathrm{fs}}=\infty, 1.6 \mathrm{Mpc}^{-1}$ and $0.8 \mathrm{Mpc}^{-1}$, respectively. At these $z_{\text {sat }}$ for $M_{s}=10^{10} M_{\odot}$ the probability $F(M, z)$ is of order 0.01 , stripped down galaxies form, and the Press-Schechter formalism breaks down. Galaxy merging requires dissipation. The "saturation" observed at $M_{s}=10^{12} M_{\odot}$ may be due to the long time required for "dry" mergers of galaxies with little gas content. In conclusion, to measure $k_{\mathrm{fs}}$, we need to compare observations with calculations at $z \gtrsim 5$, before the saturation sets in.

Note that the predictions become insensitive to $k_{\mathrm{fs}}$ for $M>M_{\mathrm{fs}}$. Therefore, to measure $k_{\mathrm{fs}}$, we verify that prediction and data are in agreement for $M>M_{\mathrm{fs}}$. For future convenience, $\log _{10}\left(M_{\mathrm{sfs}} / M_{\odot}\right) \approx \log _{10}\left(M_{\mathrm{fs}} / M_{\odot}\right)-0.63=10.5,10.9,11.5$ for $k_{\mathrm{fs}}=1.6,1.2,0.8 \mathrm{Mpc}^{-1}$, respectively.

\section{Measurements of $\boldsymbol{k}_{\mathrm{fs}}$ from Stellar Mass Distributions with $\mathrm{z} \approx 5.5$ to $\mathrm{z} \approx 8.5$}

Reference [18] presents a compilation of measured stellar mass functions for redshifts $z \approx 0$ to $z \approx 8.5$, and estimates the systematic uncertainties imposing continuity equation constraints. The measurements with $z \approx 5.5$ to $z \approx 8.5$ [19] [20] [21] are compared with calculations in Figures 8-10. From these figures we obtain the measurements of $k_{\mathrm{fs}}$ summarized in Table 1. Note that the bin centered at $z=4.5$ already shows signs of saturation at the high mass end, see Figure 11 .

Taking the Ellipsoidal Collapse model with $\tilde{v}=0.84 v$ as the preferred prediction with an uncertainty $\Delta k_{\mathrm{fs}}={ }_{-0.1}^{+0.3} \mathrm{Mpc}^{-1}$ (see Table 1), the contribution of correlated systematic uncertainties of the data obtained in Reference [18], $\pm 0.15 \mathrm{Mpc}^{-1}$,

Table 1. Measurements of the warm dark matter cut-off wavenumber $k_{\mathrm{fs}}$ obtained from Figures 8-10, assuming the validity of the Press-Schechter, Ellipsoidal Collapse with $\tilde{v}=v$, and Ellipsoidal Collapse with $\tilde{v}=0.84 v$, approximations. The total uncertainties shown include statistical uncertainties, and systematic uncertainties estimated in [18].

\begin{tabular}{cccc}
\hline$Z$ & $\begin{array}{c}k_{\mathrm{fs}}\left[\mathrm{Mpc}^{-1}\right] \\
\text { Press-Schechter }\end{array}$ & $\begin{array}{c}k_{\mathrm{fs}}\left[\mathrm{Mpc}^{-1}\right] \\
\text { Ellipsoidal collapse, } v\end{array}$ & $\begin{array}{c}k_{\mathrm{fs}}\left[\mathrm{Mpc}^{-1}\right] \\
\text { Ellipsoidal collapse, } 0.84 v\end{array}$ \\
\hline 8 & $1.10 \pm 0.30$ & $1.10 \pm 0.40$ & $0.80 \pm 0.30$ \\
$\approx 7$ & $1.10 \pm 0.30$ & $1.25 \pm 0.35$ & $0.85 \pm 0.25$ \\
$\approx 6$ & $1.10 \pm 0.30$ & $1.25 \pm 0.35$ & $0.80 \pm 0.30$ \\
\hline
\end{tabular}




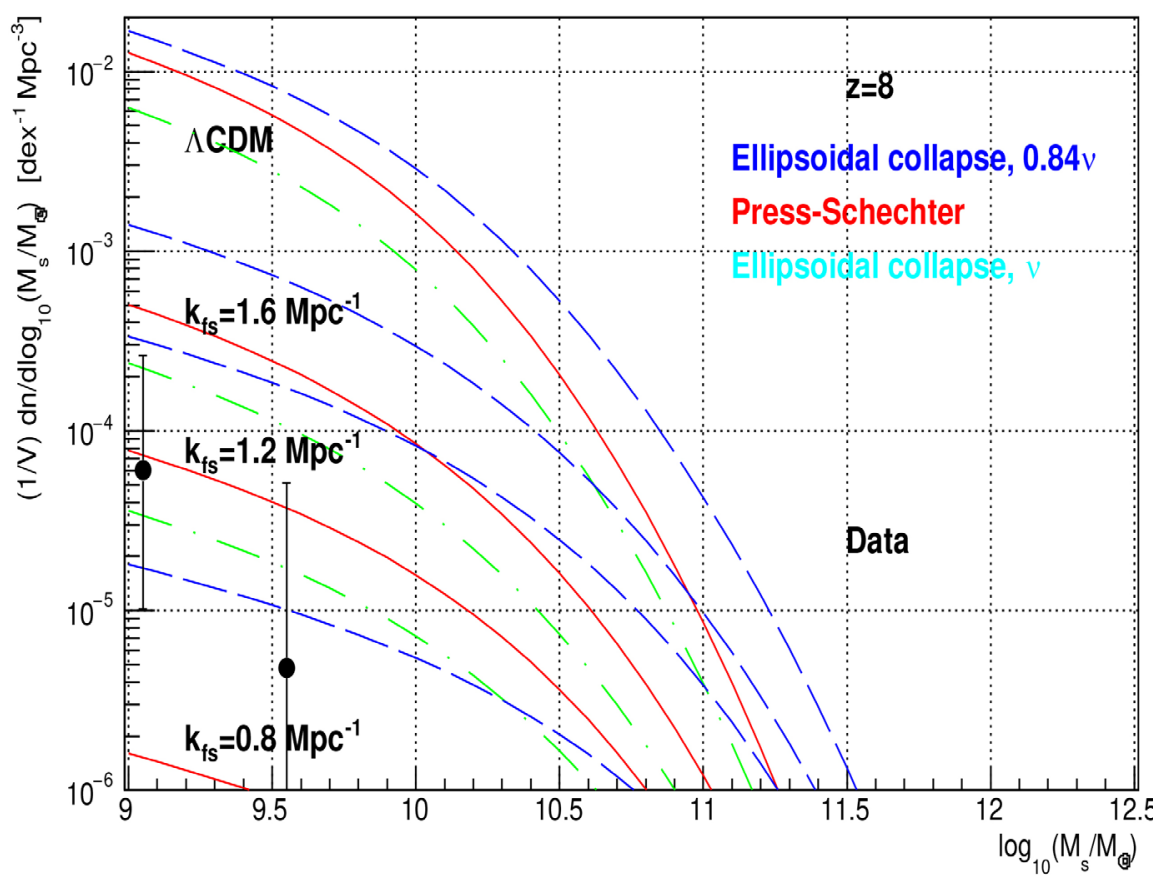

Figure 8. Calculated stellar mass functions with the Press-Schechter, Ellipsoidal Collapse with $\tilde{v}=v$, and Ellipsoidal Collapse with $\tilde{v}=0.84 v$, approximations, for $\Lambda \mathrm{CDM}$, and $\Lambda \mathrm{WDM}$ with $k_{\mathrm{fs}}=1.6,1.2$ and $0.8 \mathrm{Mpc}^{-1}$, at redshift $z=8$, compared with observations [18] [19].

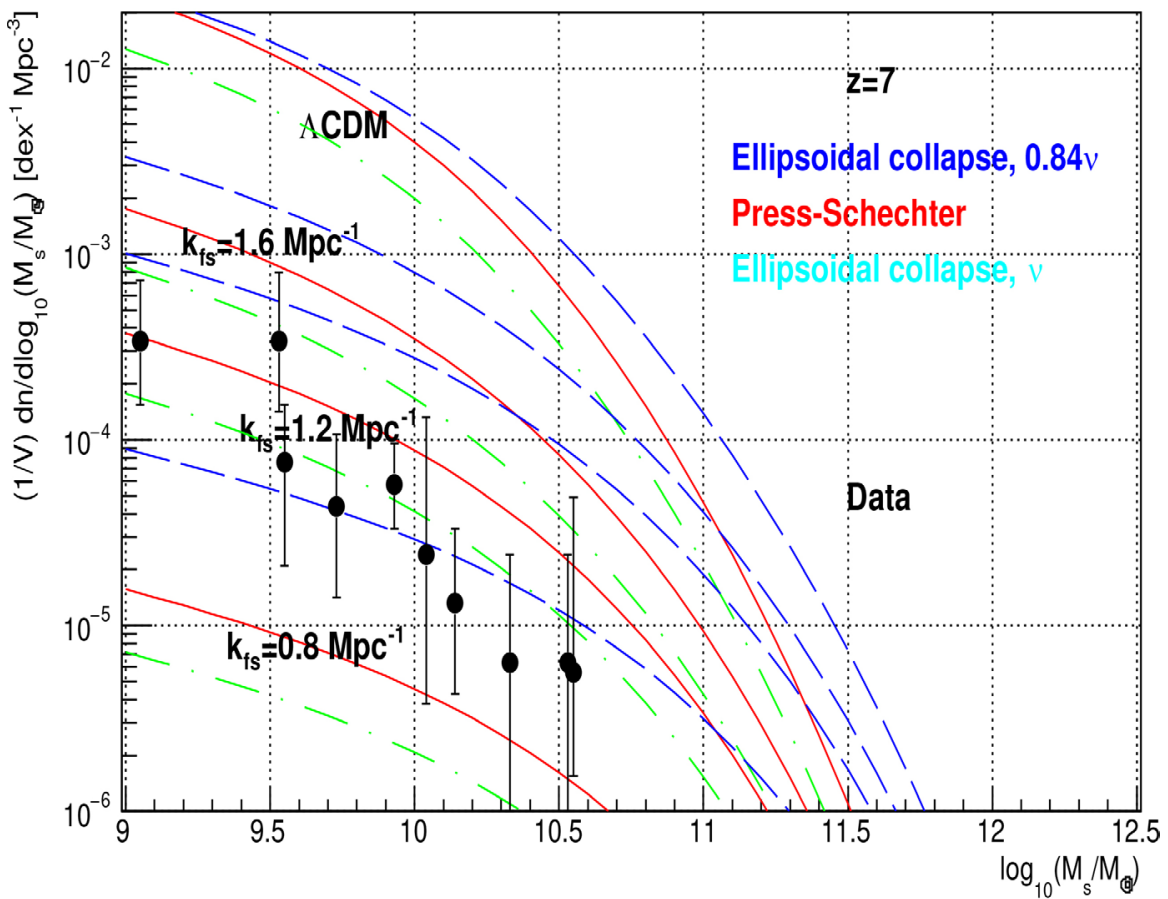

Figure 9. Calculated stellar mass functions with the Press-Schechter, Ellipsoidal Collapse with $\tilde{v}=v$, and Ellipsoidal Collapse with $\tilde{v}=0.84 v$, approximations, for $\Lambda \mathrm{CDM}$, and $\Lambda \mathrm{WDM}$ with $k_{\mathrm{fs}}=1.6,1.2$ and $0.8 \mathrm{Mpc}^{-1}$, at redshift $\mathrm{z}=7$, compared with observations [18] [19] [20]. 


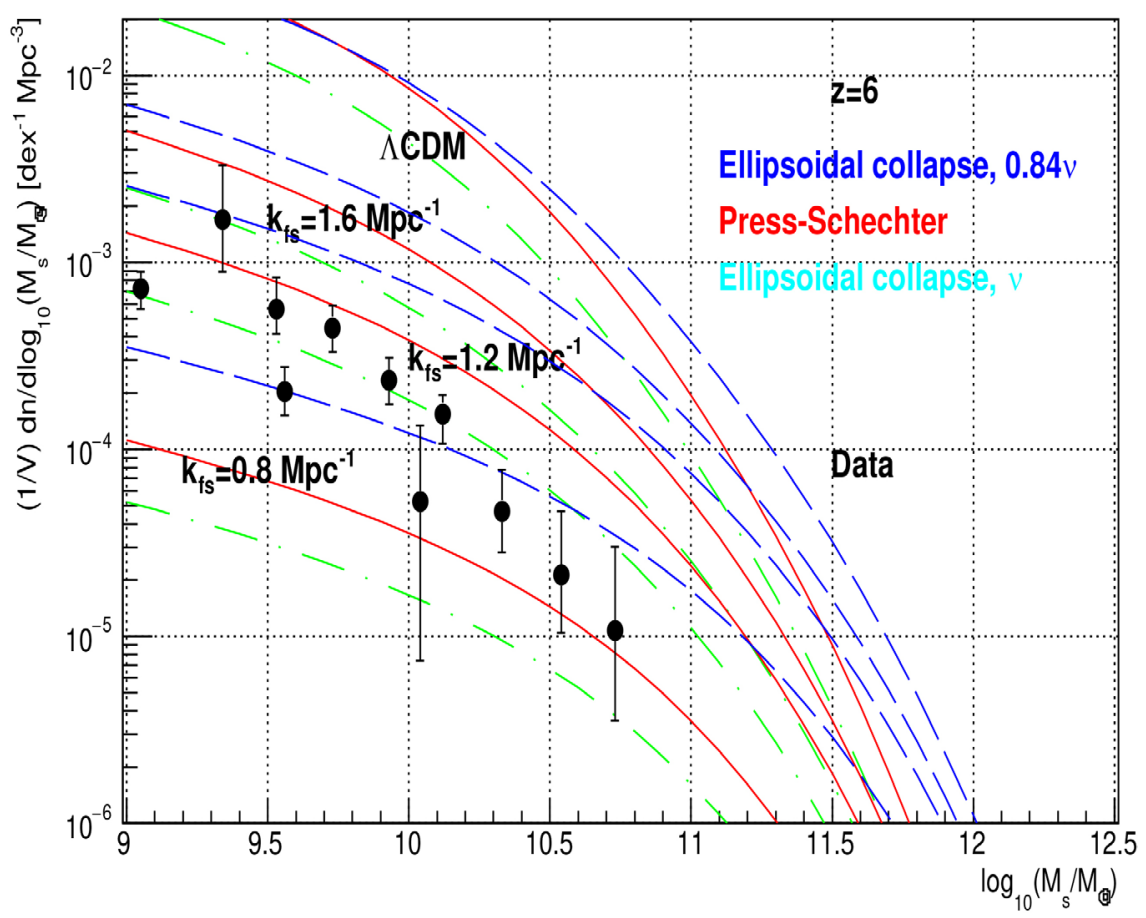

Figure 10. Calculated stellar mass functions with the Press-Schechter, Ellipsoidal Collapse with $\tilde{v}=v$, and Ellipsoidal Collapse with $\tilde{v}=0.84 v$, approximations, for $\Lambda \mathrm{CDM}$, and $\Lambda \mathrm{WDM}$ with $k_{\mathrm{fs}}=1.6,1.2$ and $0.8 \mathrm{Mpc}^{-1}$, at redshift $\mathrm{z}=6$, compared with observations [18] [19] [20].

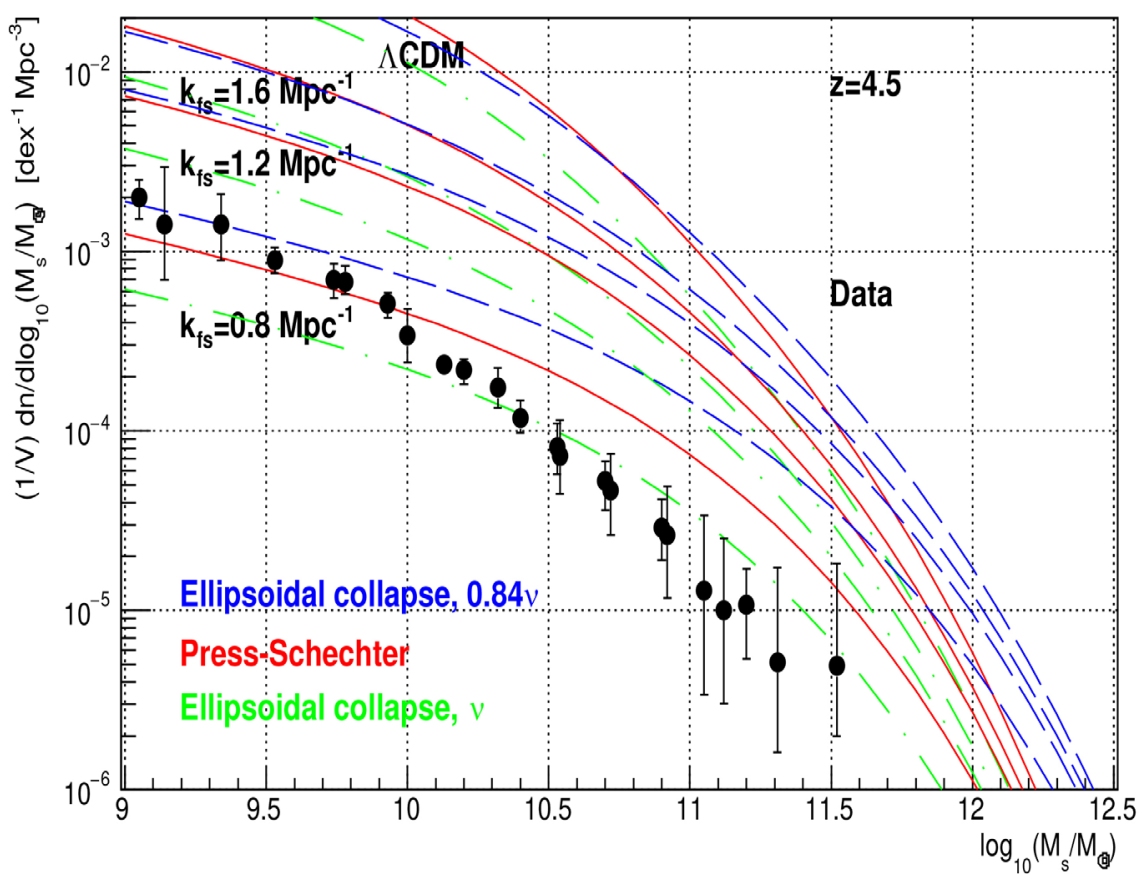

Figure 11. Calculated stellar mass functions with the Press-Schechter, Ellipsoidal Collapse with $\tilde{v}=v$, and Ellipsoidal Collapse with $\tilde{v}=0.84 v$ approximations, for $\Lambda \mathrm{CDM}$, and $\Lambda \mathrm{WDM}$ with $k_{\mathrm{fs}}=1.6,1.2$ and $0.8 \mathrm{Mpc}^{-1}$, at redshift $\mathrm{z}=4.5$, compared with observations at $z \approx 4.5$ [18] [19] [20] [21]. Note the onset of "saturation" at the high mass end (that is not understood). 
an uncertainty due to $P(k), \pm 0.2$, and statistical uncertainties, we obtain our final measurement: $k_{\mathrm{fs}}=0.90_{-0.34}^{+0.44} \mathrm{Mpc}^{-1}$. This result is insensitive to the "tail" in (3).

(Note: The present measurement of $k_{\mathrm{fs}}$ superceeds the estimate in Reference [3] that was based on data in SDSS DR15, class stellarMassFSPSGranWideDust that shows strong top down galaxy evolution, see Figure 4.)

\section{Estimate of $\boldsymbol{k}_{\mathrm{fs}}$ from Galaxy GN-z11}

The galaxy with largest spectroscopically confirmed redshift to date is GN-z11 with $Z=11.09_{-0.12}^{+0.08} \quad$ [22]. Its stellar mass is estimated to be $M_{s} \approx 10^{9} M_{\odot}$. One such galaxy was found in a comoving search volume $V=1.2 \times 10^{6} \mathrm{Mpc}^{3}$, for $\Delta z=1$. Figure 12 compares this single galaxy with expectations corresponding to the cut-off factor (3). To illustrate the effect of the cut-off factor tail, Figure 13 presents the expectations corresponding to the gaussian cut-off factor (2). From this single galaxy we obtain $k_{\mathrm{fs}} \approx 1.1 \mathrm{Mpc}^{-1}$.

\section{Conclusion}

Comparing measurements of stellar mass distributions of galaxies in the redshift range $5.5 \lesssim z \lesssim 8.5$ with expectations, we obtain the warm dark matter cut-off wavenumber $k_{\mathrm{fs}}=0.90_{-0.34}^{+0.44} \mathrm{Mpc}^{-1}$. This result is in agreement with the independent measurements obtained by fitting spiral galaxy rotation curves (demonstrating that the cut-off $k_{\mathrm{fs}}$ is due to warm dark matter free-streaming), and is consistent with the scenario of dark matter with no freeze-in and no freeze-out, see Table 2 [3] [23] [24] [25]. Detailed properties of warm dark matter can be derived from $k_{\mathrm{fs}}[3]$. The observed stellar mass functions disfavor the $\Lambda \mathrm{CDM}$ model.

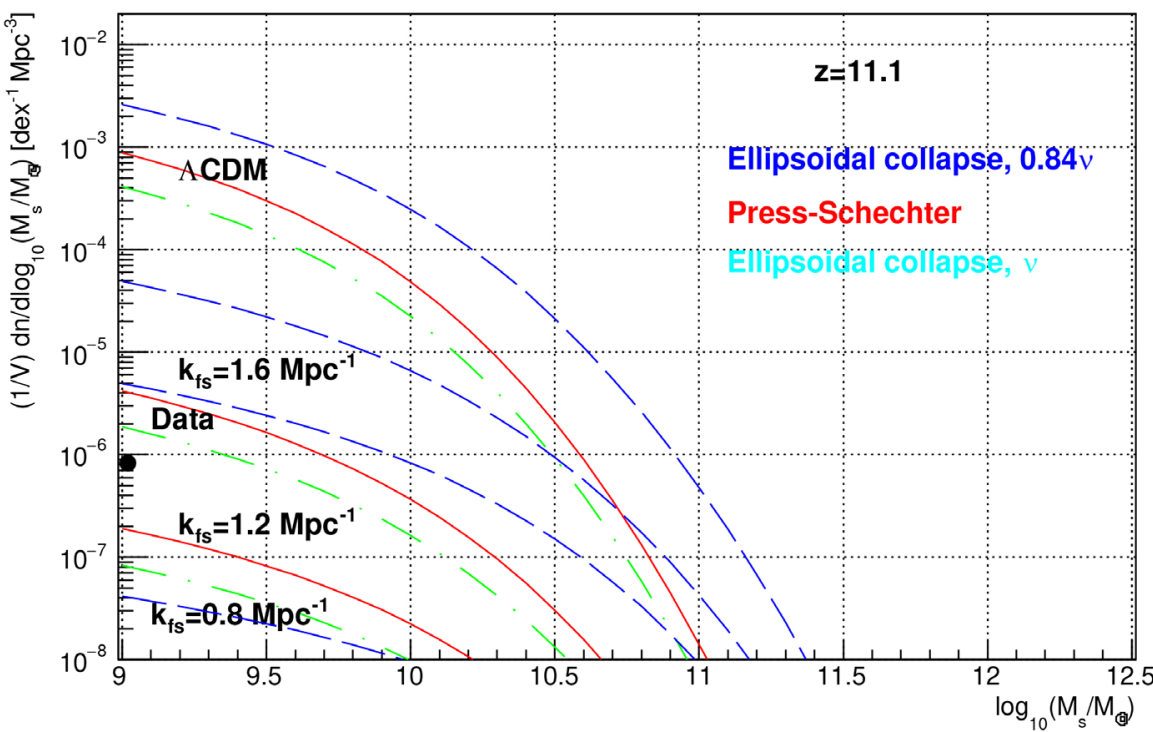

Figure 12. Calculated stellar mass functions with the Press-Schechter, Ellipsoidal Collapse with $\tilde{v}=v$, and Ellipsoidal Collapse with $\tilde{v}=0.84 v$, approximations, for $\Lambda \mathrm{CDM}$, and $\Lambda \mathrm{WDM}$ with $k_{\mathrm{fs}}=1.6,1.2$ and $0.8 \mathrm{Mpc}^{-1}$, at redshift $z=11.1$, compared with one observed galaxy GN-z11 (assuming one similar galaxy per dex) [22]. The cut-off factor is given in Equation (3). This graph obtains $k_{\mathrm{fs}}$ of the order of $1.1 \mathrm{Mpc}^{-1}$. 


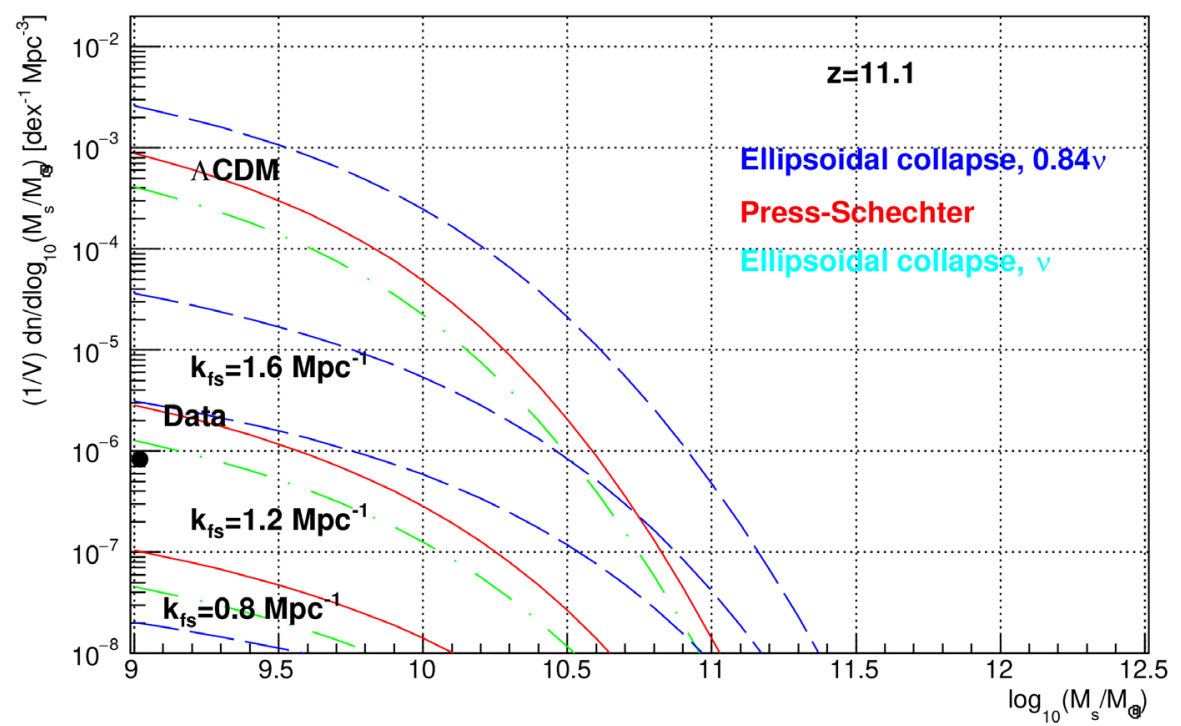

Figure 13. Same as Figure 12, but with the gaussian cut-off factor (2).

Table 2. Update of Table 2 of Reference [3]. Summary of three independent measurements of the adiabatic invariant $v_{h r m s}(1)$ [3], the expansion parameter at which dark matter particles become non-relativistic $a_{h \mathrm{NR}}^{\prime}$, the cut-off wavenumber of warm dark matter $k_{\mathrm{fs}}$, the transition galaxy mass $M_{\mathrm{fs}}$ and the mass $m_{h}$ of dark matter particles (for the case of zero chemical potential). The top (bottom) table is for fermions with $N_{f}=2$ (bosons with $N_{b}=1$ ).

\begin{tabular}{cccccc}
\hline $\begin{array}{c}\text { Fermions } \\
\text { Observable }\end{array}$ & $v_{h \mathrm{rms}}(1)[\mathrm{km} / \mathrm{s}]$ & $a_{h \mathrm{NR}}^{\prime} \times 10^{6}$ & $m_{h}[\mathrm{eV}]$ & $k_{\mathrm{fs}}\left[\mathrm{Mpc}^{-1}\right]$ & $\log _{10}\left(M_{\mathrm{fs}} / M_{\odot}\right)$ \\
\hline Spiral galaxies & $0.76 \pm 0.29$ & $2.54 \pm 0.97$ & $79_{-17}^{+35}$ & $0.80_{-0.24}^{+0.42}$ & $12.08 \pm 0.50$ \\
No freeze-in/-out & $0.81_{-0.25}^{+0.47}$ & $2.69_{-0.84}^{+1.57}$ & $75 \pm 23$ & $0.76 \pm 0.31$ & $12.14 \pm 0.52$ \\
$M_{\mathrm{s}}$ distribution & & & & $0.90_{-0.34}^{+0.44}$ & $11.93 \pm 0.56$ \\
\hline $\begin{array}{c}\text { Bosons } \\
\text { Observable }\end{array}$ & $V_{h \mathrm{rms}}(1)[\mathrm{km} / \mathrm{s}]$ & $a_{h \mathrm{NR}}^{\prime} \times 10^{6}$ & $m_{h}[\mathrm{eV}]$ & $k_{\mathrm{fs}}\left[\mathrm{Mpc}^{-1}\right]$ & $\log _{10}\left(M_{\mathrm{fs}} / M_{\odot}\right)$ \\
\hline Spiral galaxies & $0.76 \pm 0.29$ & $2.54 \pm 0.97$ & $51_{-11}^{+22}$ & $0.51_{-0.15}^{+0.28}$ & $12.66 \pm 0.50$ \\
No freeze-in/-out & $0.26_{-0.08}^{+0.16}$ & $0.88_{-0.28}^{+0.52}$ & $113 \pm 35$ & $1.26 \pm 0.50$ & $11.49 \pm 0.52$ \\
$M_{\mathrm{s}}$ distribution & & & & $0.90_{-0.34}^{+0.44}$ & $11.93 \pm 0.56$ \\
\hline
\end{tabular}

\section{Acknowledgements}

Funding for the Sloan Digital Sky Survey IV has been provided by the Alfred P. Sloan Foundation, the U.S. Department of Energy Office of Science, and the Participating Institutions. SDSS-IV acknowledges support and resources from the Center for High-Performance Computing at the University of Utah. The SDSS web site is http://www.sdss.org.

SDSS-IV is managed by the Astrophysical Research Consortium for the Participating Institutions of the SDSS Collaboration including the Brazilian Participation 
Group, the Carnegie Institution for Science, Carnegie Mellon University, the Chilean Participation Group, the French Participation Group, Harvard-Smithsonian Center for Astrophysics, Instituto de Astrofísica de Canarias, The Johns Hopkins University, Kavli Institute for the Physics and Mathematics of the Universe (IPMU)/University of Tokyo, the Korean Participation Group, Lawrence Berkeley National Laboratory, Leibniz Institut für Astrophysik Potsdam (AIP), MaxPlanck-Institut für Astronomie (MPIA Heidelberg), Max-Planck-Institut für Astrophysik (MPA Garching), Max-Planck-Institut für Extraterrestrische Physik (MPE), National Astronomical Observatories of China, New Mexico State University, New York University, University of Notre Dame, Observatário Nacional/MCTI, The Ohio State University, Pennsylvania State University, Shanghai Astronomical Observatory, United Kingdom Participation Group, Universidad Nacional Autónoma de México, University of Arizona, University of Colorado Boulder, University of Oxford, University of Portsmouth, University of Utah, University of Virginia, University of Washington, University of Wisconsin, Vanderbilt University, and Yale University.

\section{Conflicts of Interest}

The author declares no conflicts of interest regarding the publication of this paper.

\section{References}

[1] Tanabashi, M., et al. (Particle Data Group) (2018) The Review of Particle Physics. Physical Review D, 98, Article ID: 030001. https://doi.org/10.1103/PhysRevD.98.030001

[2] Drewes, M., et al. (2017) A White Paper on keV Sterile Neutrino Dark Matter. JCAP, 1701, 25.

[3] Hoeneisen, B. (2019) Simulations and Measurements of Warm Dark Matter Free-Streaming and Mass. International Journal of Astronomy and Astrophysics, 9, 368-392. https://doi.org/10.4236/ijaa.2019.94026

[4] Weinberg, S. (2008) Cosmology. Oxford University Press, Oxford.

[5] Boyanovsky, D., de Vega, H.J. and Sanchez, N.G. (2008) The Dark Matter Transfer Function: Free Streaming, Particle Statistics and Memory of Gravitational Clustering. Physical Review D, 78, Article ID: 063546. https://doi.org/10.1103/PhysRevD.78.063546

[6] Agertz, O., Teyssier, R. and Moore, B. (2009) Disk Formation and the Origin of Clumpy Galaxies at high Red-Shift. Monthly Notices of the Royal Astronomical Society, 397, L64-L68. https://arxiv.org/pdf/0901.2536.pdf https://doi.org/10.1111/j.1745-3933.2009.00685.x

[7] Bildfell, C., Hoekstra, H., Babul, A., et al. (2012) Evolution of the Red Sequence Giant to Dwarf Ratio in Galaxy Clusters out to $\mathrm{Z}=0.5$. Monthly Notices of the Royal Astronomical Society, 425, 204-221. https://arxiv.org/pdf/1202.6058.pdf https://doi.org/10.1111/j.1365-2966.2012.21426.x

[8] Press, W.H. and Schechter, P. (1974) Formation of Galaxies and Clusters of Galaxies by Self-Similar Gravitational Condensation. The Astrophysical Journal, 187, 425-438. https://doi.org/10.1086/152650

[9] Bond, J.R., et al. (1991) Excursion Set Mass Functions for Hierarchical Gaussian Fluctuations. The Astrophysical Journal, 379, 440. https://doi.org/10.1086/170520 
[10] Sheth, R.K. and Tormen, G. (1999) Large-Scale Bias and the Peak Background Split. Monthly Notices of the Royal Astronomical Society, 308, 119-126. https://doi.org/10.1046/j.1365-8711.1999.02692.x

[11] Sheth, R.K., Mo, H.J., Tormen, G. (2001), Ellipsoidal collapse and an improved model for the number and spatial distribution of dark matter haloes, Monthly Notices of the Royal Astronomical Society, 323, 1-12. https://doi.org/10.1046/j.1365-8711.2001.04006.x

[12] Ahumada, R., et al. (2019) The Sixteenth Data Release of the Sloan Digital Sky Surveys: First Release from the APOGEE-2 Southern Survey and Full Release of eBOSS Spectra.

[13] Conroy, Ch., Gunn, J. and White, M. (2009) The Propagation of Uncertainties in Stellar Population Synthesis Modeling I: The Relevance of Uncertain Aspects of Stellar Evolution and the IMF to the Derived Physical Properties of Galaxies. The Astrophysical Journal, 699, 486-506. https://doi.org/10.1088/0004-637X/699/1/486

[14] Maraston, C., Strömbäck, G., Thomas, D., Wake, D.A. and Nichol, R.C. (2008) Modeling the Color Evolution of Luminous Red Galaxies-Improvements with Empirical Stellar Spectra. https://arxiv.org/pdf/0809.1867.pdf

[15] Chen, et al. (2012) Evolution of the Most Massive Galaxies to $\mathrm{z}=0.6$. I. A New Method for Physical Parameter Estimation. Monthly Notices of the Royal Astronomical Society, 421, 314-332. https://doi.org/10.1111/j.1365-2966.2011.20306.x

[16] Guo, H., Yang, X.H. and Lu, Y. (2018) The Incomplete Conditional Stellar Mass Function: Unveiling the Stellar Mass Functions of Galaxies at $0.1<\mathrm{z}<0.8$ from BOSS Observations. https://arxiv.org/pdf/1804.01993.pdf https://doi.org/10.3847/1538-4357/aabc56

[17] Capozzi, D., et al. (2017) Evolution of Galaxy Luminosity and Stellar-Mass Functions since $\mathrm{z}=1$ with the Dark Energy Survey Science Verification Data. Monthly Notices of the Royal Astronomical Society, 1.

[18] Lapi, A., et al. (2017) Stellar Mass Function of Active and Quiescent Galaxies via the Continuity Equation. https://arxiv.org/pdf/1708.07643.pdf https://doi.org/10.3847/1538-4357/aa88c9

[19] Song, M., Finkelstein, S.L., Ashby, M.L.N., et al. (2016) The Evolution of the Galaxy Stellar Mass Function at $\mathrm{z}=4$-8: A Steepening Low-Mass-End Slope with Increasing Redshift. ApJ, 825, 5. https://doi.org/10.3847/0004-637X/825/1/5

[20] Grazian, A., Fontana, A., Santini, P., et al. (2015) The Galaxy Stellar Mass Function at $3.5 \leq \mathrm{z} \leq 7.5$ in the CANDELS/UDS, GOODS-South, and HUDF Fields. Astronomy \& Astrophysics, 575, A96. https://doi.org/10.1051/0004-6361/201424750

[21] Davidzon, I., Ilbert, O., Laigle, C., et al. (2017) The COSMOS2015 Galaxy Stellar Mass Function: 13 Billion Years of Stellar Mass Assembly in 10 Snapshots. Astronomy \& Astrophysics, 605, A70. https://doi.org/10.1051/0004-6361/201730419

[22] Oesch, P.A., et al. (2016) A Remarkably Luminous Galaxy at $\mathrm{z}=11.1$ Measured with Hubble Space Telescope GRISM Spectroscopy.

[23] Hoeneisen, B. (2019) A Study of Dark Matter with Spiral Galaxy Rotation Curves. International Journal of Astronomy and Astrophysics, 9, 71-96. https://doi.org/10.4236/ijaa.2019.92007

[24] Hoeneisen, B. (2019) A Study of Dark Matter with Spiral Galaxy Rotation Curves. Part II. International Journal of Astronomy and Astrophysics, 9, 133-141. https://doi.org/10.4236/ijaa.2019.92010

[25] Hoeneisen, B. (2019) The Adiabatic Invariant of Dark Matter in Spiral Galaxies. International Journal of Astronomy and Astrophysics, 9, 355-367. 